\title{
Survey Paper of Energy Efficient Data Aggregation Protocol in Wireless Sensor Network
}

\author{
Chanchal Sharma \\ Research Scholar (M.Tech) \\ Krishna Engineering \\ Collage Ghaziabad, INDIA
}

\author{
Vishwajeet Pattanik \\ Assistant Professor \\ Krishna Engineering \\ Collage Ghaziabad, INDIA
}

\begin{abstract}
A wireless sensor network(WSN) is usually composed of a large collection of small autonomous sensor device that can sense environmental condition about the ambient environment. The main task of wireless sensor node is a communicate together by many wireless strategies. In this communication strategies need fast data access in wireless sensor network(WSN), as resource constrained is a main issue in wireless sensor networks applications. For this solution administered routing protocol. Routing protocol are in charge of discovering and maintaining the route in the network. Routing protocol with low energy consumption play a very important role in prolonging the lifetime of sensor network. Cluster based routing protocols have proven to be effective in network topology management, energy minimization and data aggregation and so on. In this paper we present various cluster- based routing protocols and merits and limitations of protocols.
\end{abstract}

\section{Keywords}

Wireless sensor network, cluster-based routing, data aggregation, energy minimization.

\section{INTRODUCTION}

Wireless sensor network are special kind ad-hoc network, having abilities of sensing, processing and wireless connectivity. Wireless sensor network (WSN)contains hundreds or thousands of sensor nodes have the ability to communicate among each other, have limited energy source, energy constrained and bandwidth. The sensors coordinate among themselves to form a communication network such as a single multi-hop network or a hierarchical organization with several clusters and cluster heads to collect the data to sink node in the WSN. Because of limited computing resources of the sensor parents a major challenges for routing protocol and algorithms. Considerable techniques are required to make them energy efficient that would increase the life-time of a WSN [1][2][3][6]. Since sensor nodes are energy constrained, it is inefficient for all the sensors to transmit the data directly to the base station. Data aggregation is the global process of gathering and routing information through a multi hop network with the objective of reducing resource consumption (in particular energy) and prolong the network lifetime in WSNs[4].

In this paper we present the cluster based routing protocols for energy efficient data aggregation in WSNs. In section 2 presents the data aggregation. In section 3 presents the classification of routing protocols in WSNS. In section 4 presents the cluster based routing protocols in WSNs and merits and limitations of cluster based routing protocols. In section 5 presents the issues in cluster based routing protocols. In section 6 concludes the paper.

\section{DATA AGGREGATION}

The main purpose of data aggregation is to reduce the power consumption by minimizing the number of data transmission. Data aggregation is defines as the process of aggregating the data from multiple sensors to eliminate redundant transmission to the base station. All the aggregation nodes collect data from their children nodes and calculate the aggregation value. Then only the aggregated values are forwarded towards the data sink. The aggregate value may be average, maximum (minimum), summation, etc. which is calculated according to the application requirements. Data generated from neighboring sensors is often redundant and highly correlated. In addition, the amount of data generated in large sensor networks is usually enormous for the base station to process. Data aggregation usually involves the fusion of data from multiple sensors at intermediate nodes and transmission of the aggregated data to the base station.

The sensors periodically sense the data, process it and transmit it to the base station. The frequency of data reporting and the number of sensors which report data usually depends on the specific application. The efficiency of data aggregation algorithm depends on the correlation among the data generated by different information source (sensor units). A correlation can be either spatial or temporal. Aggregation is defines the measure of reduction in the communication traffic due to the aggregation. The most important ingredient for aggregation is a well designed routing protocol, classified as classic (address centric) routing protocols typically forward data along the shortest path to the destination and Data centric routing protocols forward data based on the packet content and choose the next hop in order to promote in-network aggregation and minimize energy expenditure [4][5][6].

\section{ROUTING PROTOCOLS IN \\ WIRELESS SENSOR NETWORK}

\subsection{Design challenges of routing protocols in WSNs}

Routing protocol [7][8] in WSNs are responsible for discovering and maintaining energy efficient routes in the networks, in order to make communication reliable and efficient. Due to the limitations in the kind of network, the main aim of routing protocol design is extending the network life time by keeping the sensors alive as much as possible. This issue results in keeping the network connected for a long period of time. There are some challenging factors which are important in designing routing protocols. These are given as:

\subsubsection{Node Deployment}

Deployment is very application dependent and affects the performance of the routing protocols. It can be manual or randomized [8][9]. In the first strategy, the nodes are manually placed and data is routed through predestined paths. 
In manual deployment, coverage of area is satisfied with careful choice of node density. Although, this is good choice when nodes are costly and their operations are influenced by their locations, it is not good for harsh environment [10]. On the other hand, in random deployment, the nodes are scattered arbitrarily. If the application is related to event detection, then it is efficient to have a random node deployment to get effective results [11][12].

\subsubsection{Energy consumption}

The main aim of routing protocols is to convey data among sensors and sink in efficient manner. Each sensor node consumes energy in sensing, processing, receiving and transmitting information [13]. Among these data transmission is the most energy consuming task [14]. Since, the sensor nodes have limited energy resource, energy depletion of some nodes results in great topology and network connectivity changes, reorganization of network and finding new routes. So, there is a need to design routing protocols that can accommodate the tradeoff between energy optimization and accuracy [14][15].

\subsubsection{Nature of node}

In WSN, the nodes that are scattered over the environments can be either homogeneous or heterogeneous. Homogeneous nodes have the same capabilities such as, rang of transmission, battery life, and processing capacity while heterogeneous nodes have different capabilities [8]. The majority of network architecture assumes that the sensor nodes are stationary. However, mobility of base station as well as nodes is necessary in several applications [17].

\subsubsection{Coverage}

In WSNs, each node prevail a certain view of the environment. A given sensor's view of the environment is limited both in range and in accuracy. Hence coverage area is essential design issue [14].

\subsubsection{Scalability}

The number of nodes deployed in the field may be variable i.e. few numbers to few thousands. The routing protocol be required to be able to work with massive amount of nodes [8][14]. When the number of nodes is extensive, it is infeasible that each node maintain a global knowledge of network topology.

\subsubsection{Quality of service (QoS)}

The routing protocol should be able to provide certain level of QoS that is required by the application. The QoS parameters can be bandwidth, delivery delay, throughput, jitter etc [18]. For instance, target detection and tracking applications required high throughput [19].

\subsubsection{Application}

The routing protocols are very application specific. In other word, different scenario or network environment need different routing protocols. From the application's viewpoint, data can be collected from the environment using various methods such as, time driven, event driven, and query driven methods. In time driven methods, the sensor nodes send their data periodically to BS or Gateways. In event driven methods, sensors nodes report the collected data when the event occurs. Eventually, in query driven methods, the BS request the data from the nodes and send a query.

\subsection{Classification of routing protocols in WSNs}

In WSNs, the network layer is used to implement the routing of incoming data. In multi-hop networks, the source node cannot reach the sink directly. So, intermediate nodes have to relay their packets. The implementation of routing tables gives the solution. WSN routing protocols can be classified into three ways, according to the way of establishing the routing paths, according to the network structure, according to the protocol operation, according to the initiator of communication, and according to how a protocol selects a next hop on route of forwarded message.

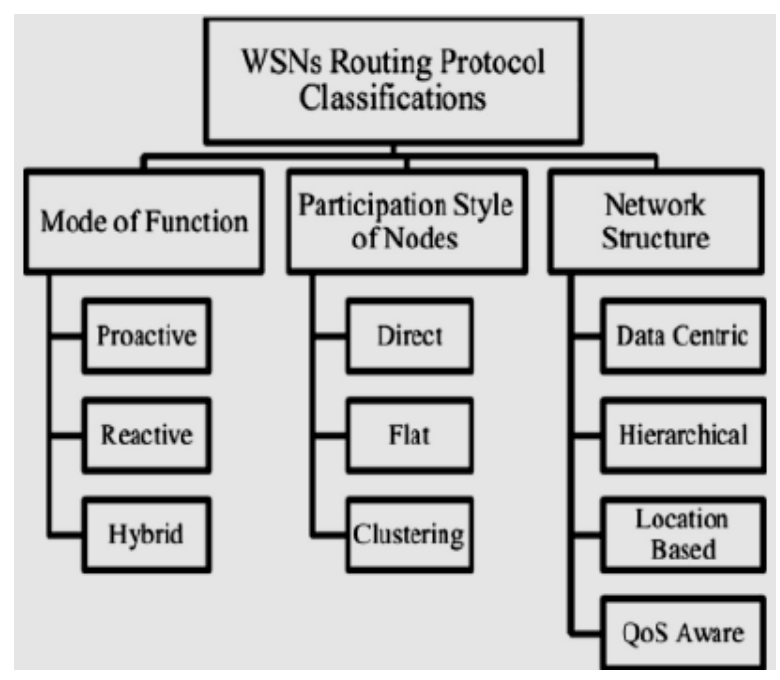

Fig 1: Taxonomy of routing protocols in WSNs

The network structure based routing protocols are categorized as: flat based, hierarchal based (cluster based), and direction based routing protocols. In flat based routing, every sensor node plays same role. While, in cluster based routing, sensor nodes have different roles. So, when networks salability and efficient communication is needed, cluster based routing is the best choice.

\section{CLUSTER BASED ROUTING PROTOCOLS IN WSNS}

The cluster based routing [20][21][22] is energy efficient method in which nodes those having high energies are arbitrarily selected for processing and sending data while nodes those having low energies are used for sensing and sending information to the cluster heads $(\mathrm{CHs})$. This property of cluster based routing contributes to the scalability, lifetime maximization, and energy minimization. The cluster based routing protocols plays a pivotal role in achieving application specific goals. The cluster based routing protocols are classified into three broad categories as in fig 2: block cluster based, grid cluster based, and chain cluster based routing protocols.

The popular block cluster based routing protocols are: LEACH, HEED UCS, EECS, CCM, TEEN, LEACH-VF etc. The popular grid cluster based routing protocols are: PANEL, GAF, TTDD, SLGC etc. the popular chain cluster based

routing protocols are: PEGASIS, CCS, TSC etc. 


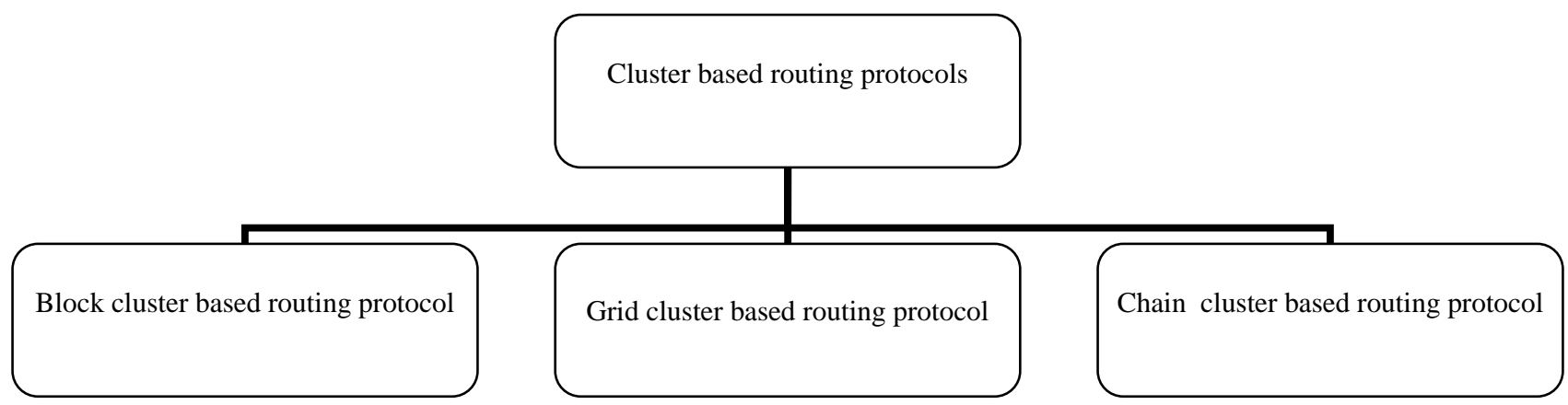

Fig 2: classification of cluster based routing protocol

\subsection{Merits and limitations of block cluster based routing protocols}

These are the following merits and limitations of block cluster based routing protocol:

LEACH algorithm merits:

- $\quad$ Each node has equal chance to become cluster head but cannot be selected as cluster head in subsequent round so load is shared between nodes

- LEACH uses TDMA so it keeps CHs from unnecessary collisions

Limitations:-

- $\quad$ LEACH use single hop communication so it can not used in large scale networks

- $\mathrm{CHs}$ are elected on the basis of probability so uniform distribution cannot be ensured and it cannot provide load balancing

HEED algorithm merits:-

- Fully distributed routing scheme

- HEED achieves load balancing and uniform $\mathrm{CH}$ distribution

- HEED achieves high energy efficiency and scalability by communicating in multi-hop way

Limitations:-

- Unbalancing energy consumption due to more $\mathrm{CH}$ generation

- Massive overhead due to multiple rounds

- Additional overhead due to several epochs

UCS algorithm merits:-

- Nodes in cluster can be variable

- UCS is bi-layered model and two-hop inter cluster communication

Limitations:-

- It is limited by assumptions that $\mathrm{CHs}$ are predetermined as well as network is not homogeneous

- Residual energy of node is not considered and not sufficient for large range networks

EECS algorithm merits:-
- $\quad$ EECS constructs more balanced network in term of energy consumption and communication load

- Use dynamic sizing of clusters

Limitations:-

- Lot of overhead due to global information for communication

- $\quad$ Single hop communication consume lot of energy

CCM algorithm merits:-

- Less energy consumption compared with LEACH Limitations:-

- Chain head selection criterion

LEACH-VF algorithm merits:-

- $\quad$ Solve the problem of area with overlapped sensing coverage and sensing hole

- In LEACH-VF some nodes can be moved to coverage inside the cluster are

Limitations:-

- $\quad$ Poor energy efficiency

- Load balancing is not up to the mark

TEEN algorithm merits:-

- Data transmission can be controlled by varying two thresholds

- Well suited for time critical applications

Limitations:-

- Whenever thresholds are not meet, the node will not communicate

- Data may be lost if $\mathrm{CHs}$ are not able to communicate with each other

\subsection{Merits and limitations of grid cluster based routing protocols}

These are the following merits and limitations of grid based routing protocols:

PANEL algorithm merits:-

- PANEL is energy efficient that ensure load balancing and long network life time

- Supports asynchronous applications 
Limitations:-

- Clusters are predetermined

- To determine geographic position information, special conditions are needed, which is not always available

GAF algorithm merits:-

- GAF increase the network lifetime by saving energy

- $\quad$ Routing fidelity is maintained

Limitation:-

- Large traffic injection and delay is not predictable

TTDD algorithm merits:-

- Resolve the numerous mobile sinks and moving problem of sink in large scale WSNs

- Suitable to event detecting WSNs among irregular data traffic

Limitations:-

- $\quad$ Large latency

- Low energy efficiency

SLGC algorithm merits:-

- Lower energy consumption in SLGC compared to $\mathrm{LEACH}$

Limitation:-

- Large overhead due to complex data communication

\subsection{Merits and limitations of chain cluster based routing protocols}

These are the following merits and limitations of chain cluster based routing protocols:

PEGASIS algorithm merits:-

- Energy load is distributed uniformly

- Reduce overhead due to dynamic cluster formation

- Decrease number of data transmission

Limitations:-

- Long delay cause a node to become bottleneck

- Network is not very scalable

- Not suitable for time varying topologies

CCS algorithm merits:-

- Energy consumption is reduce

- $\quad$ Reduce data flow from BS in CCS

Limitations:-

- Unbalanced energy consumption

- $\quad$ Large delay due to long chain

TSC algorithm merits:-
- TSC reduce redundant data transmission in network in network by breaking long chains into smaller chains

Limitation:-

- Node distribution in unbalanced

\section{ISSUES IN CLUSTER BASED ROUTING PROTOCOLS}

The much research work has been done to query the drawbacks of clustering techniques and to improve the individuality of cluster based routing methods but there are still several issues to be addressed for the efficient use of cluster based routing techniques. The some open issues need to be addressed are:

- Calculation and selection of cluster heads(CHs): $\mathrm{CH}$ is utility of computation and communication energy model for the cluster schemes. If the multi hop scheme is used then $\mathrm{CHs}$ count supposed to be revised. The performance and availability of adjacent $\mathrm{CHs}$ is an important factor for relaying the data of clusters. Consequently, role in rotation of adjacent clusters must be considered as dominant factor in selection process of $\mathrm{CHs}$

- Scalability: In a few large scale deployments, it is usually desirable to enlarge the monitoring area amid new nodes. So, careful observations are required to check the adaptability and scalability of clustering techniques.

- Topology of network: Topology changes owed to territorial circumstances in realization of WSNs. So, it is important to focus on the strength of clustering methods.

- Fault tolerance: Transient fault management owed to temporal link failures desires much more attention.

- Redundancy management: Minimizing the use of massively redundant nodes for building trustworthy and proficient relay backbone. The combined data of $\mathrm{CHs}$ relayed to BS must be investigated further.

\section{CONCLUSION}

Data aggregation is one of the key techniques to solve the resource oriented problem in WSN. WSNs routing protocols with purpose to find the path to save energy and established reliable data transfer method from source to destination. This paper presents an overview of different cluster based routing protocols in wireless sensor networks, in this paper focus the merits and limitations of cluster based routing protocols. According to the merits and limitations of cluster based routing protocols, it is clear that these protocols are useful in performance improvement of wireless sensor networks. This paper will be very useful for the future research in development, modification or optimization of routing algorithms for WSNs.

\section{REFERENCES}

[1] Wen-Wen Huang, Ya-Li Peng,Jian Wen, Min Yu, "Energy-Efficient Multi-hop Hierarchical Routing Protocol for Wireless Sensor Networks", 2009 International Conference on Networks Security, Wireless Communications and Trusted computing. 
[2] Abid Ali Minhas, Fazl-e-Hadi, Danish Sattar, KashifMustaq and S. Ali Rizvi,“ Energy efficient Multicast Routing Protocols for Wireless Sensor Networks".

[3] Jamal N. Al-Karaki, Ahmed E. Kamal,“ Routing Techniques in Wireless Sensor networks: A Survey”.

[4] V. Akila1, Dr. T. Sheela,“ Overview of Data Aggregation Based Routing Protocols in Wireless Sensor Networks", 2013 International Journal of Emerging Technology and Advanced Engineering.

[5] Bhaskar Krishnamachari, Deborah Estrin, Stephen Wicker,"The Impact of Data Aggregation in Wireless Sensor Networks",

[6] Ramesh Rajagopalan, Pramod K. Varshney,"Data aggregation techniques in sensor networks: A Surevy" 2006,Electrical Engineering and Computer Science.

[7] B. Krishnamachari, D. Estrin, S. Wicker, "Modelling data centric routing in wireless sensor networks", in proceedings of IEEE INFOCOM, New York, NY, June 2002.

[8] Al-karaki, J.N., and A.E Kamal, "Routing Techniques in wireless sensor networks: a survey" , IEEE Wireless Communication 11:6-28, 2004.

[9] Yick, J., Biswanath, M., Ghosal, D., "Wireless Sensor Network Survey" , Computer Networks, vol, 52(12): 2292-2330, 2008.

[10] W.B. Heinzelman, A.P. Chandrakasan, H. Balakrishnan, "Application Specific protocol architecture for wireless micro sensor networks", IEEE Transactions on Wireless Networking, 2002.

[11] Mauri, K., Hannikainen, M., Hamalainen, T., "A Survey of application distribution in wireless sensor networks", EURASIP journal on Wireless Communications and Networking, vol. 2005:pp. 774-788, 2005.

[12] P. Kumar, M.P. Singh, and U. S. Triar, "A Review of Routing Protocols in Wireless Sensor Network",
International Journal of Engineering Research \&Technology (IJERT), vol. 1, no. 4, pp. 1-14, 2012.

[13] Romero, Kay, Friedemann Mattern, "The Design Space of Wireless Sensor Networks", IEEE Wireless Communications 11 (6): 54-61, 2004.

[14] Handed, M., D. Zhongliang, "Design challenges and Comparative analysis of cluster based routing protocols used in wireless sensor networks for improving network life time", Adv. Inform. Service Sci. 4:450-459, 2012.

[15] Akyildiz, I.F., Varun, M.C., "Wireless Sensor Networks", John Wiley \& Sons, 2010.

[16] Cordiero, C.M., Agarwal, D.P., "Ad Hoc and Sensor Networks: Theory and Applications", World Scientific, Singapore, 2011

[17] Ye, F., Lou, H., Cheng, J., Lu, S., Zhang, L., “A Two tier data dissemination model for large Scale wireless sensor networks", in Proceedings of ACM/IEEE Mobicom, 2002.

[18] Murthy, C.S.R., Manoj, B.S., "Ad Hoc Wireless Networks: Architecture and Protocols", Printice-Hall, New Jersey, USA, 2004.

[19] Dargie, W., Poellabauer, C., "Fundamental of Wireless Sensor Networks: Theory and Practice", John Wiley \& Sons, New York, USA, 2010

[20] Ameer Ahmad Abbasi, Mohamed Younis, "A Survey on Clustering algorithms for wireless sensor networks", Computer Communication 30 (2007) 2826-2841.

[21] X. Liu, "A Survey on Clustering Routing Protocols in Wireless Sensor Networks", Sensors, vol. 12 pp. 11113 $11153,2012$.

[22] S. Naeimi, H. Ghafghazi, C.O. Chow, and H. Ishi, "A Survey on the taxonomy of cluster-based routing protocols for homogenous wireless sensor networks", Sensors, vol. 12, no. 6, pp. 7350-7409, 2012. 